\title{
Electrochromically switched, gas-reservoir metal hydride devices with application to energy-efficient windows
}

\author{
André Anders, Jonathan L. Slack, and Thomas J. Richardson \\ Lawrence Berkeley National Laboratory \\ Berkeley, California
}

\begin{abstract}
Proof-of-principle gas-reservoir MnNiMg electrochromic mirror devices have been investigated. In contrast to conventional electrochromic approaches, hydrogen is stored (at low concentration) in the gas volume between glass panes of the insulated glass units (IGUs). The elimination of a solid state ion storage layer simplifies the layer stack, enhances overall transmission, and reduces cost. The cyclic switching properties were demonstrated and system durability improved with the incorporation a thin Zr barrier layer between the MnNiMg layer and the Pd catalyst. Addition of $9 \%$ silver to the palladium catalyst further improved system durability. About 100 full cycles have been demonstrated before devices slow considerably. Degradation of device performance appears to be related to Pd catalyst mobility, rather than delamination or metal layer oxidation issues originally presumed likely to present significant challenges.
\end{abstract}

\section{Introduction}

The application of electrochromic devices based on tungsten oxide has spread from the common use in rear-view mirrors to electrochromic windows for vehicles and buildings [1]. While increased comfort and architectural stylishness may be driving forces in such applications, energy savings beyond those achieved with now-standard low-emissivity 
coatings are increasingly important. This is especially true given rising energy costs and the reality of global warming.

Tungsten oxide is a preferred material in conventional electrochromic windows because of its high dynamic range, high transmittance in the clear state, environmental stability, and relative ease of fabrication. While its dark blue colored state is generally accepted as pleasant, it is absorbing in nature and therefore has limitations in controlling the energy flux associated with solar illumination. The issue of high surface temperatures when operating in direct sunlight is also of concern.

Alternative, reflecting electrochromic systems are being developed since the invention of switchable mirrors based on yttrium and lanthanum hydrides [2] and various rare earth metal hydrides [3]. These include transition metal-magnesium alloy hydrides [4-9] or the SbCu-Li [10] and copper oxide [11] systems.

Metal hydride mirrors can be switched electrochromically or gasochromically. Gasochromic switching is accomplished simply by changing the gas to which the surface of the coated glass is exposed. When the gas contains hydrogen (more than $1 \%$ but less than $4 \%$ for safety reasons), the metal layer takes up hydrogen and transforms into a transparent hydride. A catalytic capping layer of palladium facilitates the hydriding process and protects the metal layer from oxidation. Upon exposure to air, hydrogen is removed from the film and the layer spontaneously returns to the reflecting metallic phase.

For window applications, gasochromic switching is an option but it requires well controlled gas exchange processes. Alternatively, and from many points of view preferably, switching can be accomplished electrochromically, i.e. by application of a small voltage that can drive the direction of electrochemical change [12]. In a conventional electrochromic system, the switchable layer is sandwiched between transparent conductors along with an ion 
storage layer and ion conducting layer. One of the difficulties is to match the ion storage capacity with the amount of hydrogen needed for maximum switching range. For this reason, as well as to simplify the full stack of layers, an electrochromic switchable mirror system was investigated here that utilizes the volume between the two glass panes of the insulated glass unit (IGU) as the hydrogen storage reservoir. In contrast to a gasochromic system, no gas transport system is needed. The gas reservoir remains unchanged with cycling, apart from small variations in $\mathrm{H}_{2}$ partial pressure with device cycling. Such a system was developed by Wittwer et al. [13] for use in tungsten oxide electrochromic windows.

In this contribution we describe the basic design of the gas-reservoir transition metal hydride switchable mirror as well as prototype device fabrication and testing. We report on the switching performance and durability, and outline ways to improve the system that may lead to a fully functional electrochromic window with superior energy savings characteristics.

\section{Device design, fabrication, and testing}

The all-solid-state switchable mirror device is produced my magnetron sputtering of a layer system on glass.

Figure 1 shows the order of the layers in the stack. The first transparent conducting oxide (TCO) layer on glass was ITO (indium tin oxide, about $90 \mathrm{~nm}$ ) with a sheet resistance of $R_{\mathrm{s}}=15 \Omega$. Glass coated with ITO (or other TCOs) is available commercially, making it reasonable to start fabrication with precoated glass.

Sample preparation was critical. Particles of a size of the order of the layer stack may cause an electrical short between the switching metal layer and the counter electrode. Having no particles is a challenge in the absence of a clean room, but the issue has been solved with a 
strict protocol of substrate cleaning under a flow hood and careful transfer to and from the chamber.

Each glass pane (73 mm x $73 \mathrm{~mm}$ ) was segmented into four quadrants, allowing each multilayer deposition to yield four devices (Fig. 2) labeled A1, A2, B1 and B2. The motivation for making four devices was two-fold: (i) if a particle shorted one device, the remainder of the system may still give us usable results; and (ii), through application of masks, certain variations of one or more variable can be studied within one fabrication procedure.

The first layer deposited in our chamber was a $50 \mathrm{~nm}$ MnNiMg layer produced by codeposition from $\mathrm{Mg}$ and $\mathrm{Mn}_{75} \mathrm{Ni}_{25}$ alloy targets. The $\mathrm{MnNi}$ alloy was chosen as a compromise between the superior color neutrality and overall transmittance of $\mathrm{Mg}_{3} \mathrm{MnH}_{7}$ as compared to $\mathrm{Mg}_{2} \mathrm{NiH}_{4}$, and the ease of fabrication of the $\mathrm{MnNi}$ alloy as compared to pure Mn. The composition of the MnNiMg films was 8:3:89 based on deposition rate calibration, with the numbers rounded to full percents. In early experiments, the composition of some selected films was checked by Rutherford backscattering (RBS). The exact composition is not critical for the performance reported here although we found that films with Mg content between 80\% and 93\% performed well. With decreasing Mg content, switching speed increased and the upper end of the transmittance range decreased.

A $\mathrm{Zr}$ barrier layer is then deposited to minimize diffusion of Pd (from the following catalyst layer) into the MnNiMg layer. An additional function is to protect the MnNiMg layer from oxidation: the very thin film of $\mathrm{Zr}$ will oxidize readily (enthalpy of $\mathrm{ZrO}_{2}$ formation is $1100.6 \mathrm{~kJ} / \mathrm{mol}$ [14]). Yet another effect is on the growth of the following layer, catalyst layer \#1, which is either pure Pd or a co-sputtered alloy of $\mathrm{Ag}_{9} \mathrm{Pd}_{91}$. Should the $\mathrm{Zr}$ be oxidized to $\mathrm{ZrO}_{\mathrm{x}}$, or even stoichiometric $\mathrm{ZrO}_{2}$, the catalyst layer is likely to grow in island mode, as will be shown. 
Following catalyst layer \#1 is the ion conduction layer, a relatively thick layer of $\mathrm{ZrO}_{2}$. It needs to provide a low impedance path for ionic hydrogen to shuttle from the catalyst layer \#2 to the metal alloy layer and back. The exact mechanism of transport is not known but it is believed that protons are transported, and that $\mathrm{OH}$ groups in the material facilitate such transport. Therefore, sputtering was done with an $\mathrm{Ar} / \mathrm{O}_{2} / \mathrm{H}_{2} \mathrm{O}$ mixture for a number of devices, where the $\mathrm{Ar}$ and $\mathrm{O}_{2}$ flows were controlled through mass flow controllers and the $\mathrm{H}_{2} \mathrm{O}$ vapor was introduced by bubbling a portion of the argon gas through temperature-controlled water: the resulting film may be described as $\mathrm{ZrO}_{2} \cdot \mathrm{H}_{2} \mathrm{O}$. In addition to facilitating ion transport to and from the MnNiMg alloy layer, $\mathrm{ZrO}_{2}$ must electronically isolate the switchable metal layer from the ITO counterelectrode. To minimize the risk of shorts the thickness was increased from $0.3 \mu \mathrm{m}$ in initial fabrications to about $1 \mu \mathrm{m}$ in later tests.

The ITO counterelectrode layer $(140 \mathrm{~nm})$ was sputtered from an ITO target in an $\mathrm{Ar} / \mathrm{O}_{2}$ atmosphere, with $\mathrm{Ar}: \mathrm{O}_{2}$ flow ratios yielding a 2\% oxygen gas feed into the chamber.

To better facilitate the transport of hydrogen from the gas phase to the solid electrolyte (ion conductor), additional catalyst layers \#2 and \#3 were used, as indicated in figure 1. The optimum thickness and necessity of those layers is a subject of ongoing study. The multilayer coating was completed by depositing a gold bus bar along two edges of each device. The bus bars are the means to make contact to the counterelectrode when fabricating the IGU.

There was no intentional heating of the substrate throughout deposition. Given the low sputtering power of the deposition processes used here, the substrate temperature was essentially only slightly higher than room temperature.

All sputtering processes were done in one sputtering chamber without exposing the layers at any time to air: mask exchange was done through a differentially pumped load-lock system. A base pressure in the high $10^{-8}$ Torr $\left(10^{-6} \mathrm{~Pa}\right)$ region was obtained by cryopumping. 
The partial pressures during sputtering were monitored with a differentially pumped residual gas analyzer.

The sputtering power was applied as direct current (DC) except for the relatively thick film of $\mathrm{ZrO}_{2}$, which was sputtered from 2" magnetrons in dual magnetron configuration, powered by a $40 \mathrm{kHz}$ AC power supply. The deposition rates and film thicknesses were calibrated via step measurements performed with a Dektak IIA profilometer.

After completion of deposition, the glass pane with the four devices was removed from the chamber and mounted in a small IGU equipped with sets of suitable contacts and gas ports (Fig. 3). Voltages and currents as well as photopic transmittances were recorded for all four devices using a multichannel electrochemical tester (Arbin Instruments). A cycle testing protocol defined the applied voltages, a maximum allowable current, and the maximum time before polarization reversal. As a rule, the polarization was switched when the devices reached a preset transmission setpoint, which was typically $20 \%$ for the transmitting state and $3 \%$ for the reflecting state. Should the device not have reached those setpoints within 15 min (later we used $30 \mathrm{~min}$ ), the polarity was switched anyway. The low switching speed in those cases is evident by the apparent smaller switching range, appearing as a reduction of the attainable upper or lower transmittance, depending on the direction of switching speed that has become slower.

Reflectance in the metallic and transmitting states were measured using an Ocean Optics spectrometer with integrating sphere.

Selected layers or devices were characterized by optical and scanning electron microscopy, impedance spectroscopy, and transmission electron microscopy.

\section{Results}


The typical open circuit potential (OCP) varied between 4 and $500 \mathrm{mV}$, depending on the configuration of the three catalytic layers. A near-zero OCP is indicative of an electric short or otherwise defective device. Exposure of the device surface to hydrogen-containing gas could lead to spontaneous switching in cases where the $\mathrm{ZrO}_{2}$ was porous enough to allow molecular hydrogen to reach the palladium-capped metal alloy. This gasochromic switching $[4,5,9]$ is undesired in the electrochromic device system with the hydrogen-containing gas being permanently present.

When the stack is polarized at $1.0 \mathrm{~V}$ (top ITO/Pd layer positive), gaseous hydrogen is converted to protons, which are transported through the $\mathrm{ZrO}_{2}$ electrolyte to the palladium layer on the magnesium alloy surface. Here they are reduced to neutral hydrogen, which is consumed during hydride formation within the magnesium alloy. The speed of transition from the metal to metal hydride phase is influenced by the hydrogen partial pressure, the applied voltage, the impedance of the ion conduction layer, as well as the availability of the Pd catalyst to mediate formation of the hydride phases of $\mathrm{Mg}, \mathrm{Mn}$, and $\mathrm{Ni}$.

When the polarity of the applied potential is reversed to $-2.2 \mathrm{~V}$, hydrogen is depleted from the catalyst \#1 layer, producing conditions favorable to the dehydriding of the metal layer and return to the reflective metallic state. Hydrogen driven back up through the device stack ultimately returns to the gas phase.

Each of the layers in the device stack has a certain function that has been studied by targeted variations of the individual components. Fig. 4 gives examples of switching in two similar devices; here, one of them does not have the final catalyst layer (top, \#3). Both devices cycled satisfactorily for the first 20 hours after which they noticeably slow, though in different ways. While device A1, without the top layer, tends to switch quickly to the metallic, reflecting state, the conventional full stack device A2 switches relatively rapidly to the 
hydrided, transmitting state and only slowly back to the metallic state. In all cases, the switching is allowed to proceed only for 30 minutes per direction; device A1 is not reaching the desired setpoint of the transmitting state, while A2 is not reaching the desired setpoint of the reflecting state.

Another topic of research was the need to hydrate the electrolyte (ion conductor) during deposition and to maintain hydration during cycling. Based on the general belief that water improves the ion transport properties, i.e. reduces the ion impedance, water was added during deposition as mentioned before. The resulting $\mathrm{ZrO}_{2} \cdot \mathrm{H}_{2} \mathrm{O}$ layer showed a tendency to promote spontaneous gasochromic hydriding of the metal, suggesting that the films may have cracks and voids through which molecular hydrogen could reach the catalyst layer that caps the metal alloy. Electron microscopy showed the presence of such cracks in hydrated $\mathrm{ZrO}_{2}$ films, the morphology resembling material that contracted when dried (Fig. 5, top). In this study, we reduced the water content during deposition until no water vapor was added. Of course, the residual gas in our high vacuum deposition system consists mostly of water vapor and hydrogen, and the tests showed that the residual gas apparently supplied sufficient amounts of water (and hydrogen) to fabricate a well-behaving ion conducting layer. The recently produced, best performing devices had a $\mathrm{ZrO}_{2}$ layer made without water intentionally added.

The phase transition from metal to metal hydride is accompanied by a $32 \%$ volume expansion [4], which puts strain on the system. This is especially a problem when the deposited layers are already under residual stress from the growth process, and in areas where film non-uniformity and defects promote a locally faster phase change. The situation could be aggravated if hydrogen atoms recombine and form gaseous hydrogen below the top layers of the stack. Fig. 6 shows an intriguing optical micrograph. We look through the transparent 
counterelectrode (ITO) that is partially delaminated. The ITO has the typical relaxation pattern of a film whose intrinsic compressive stress is relieved by delamination.

To have a closer look at the Pd film growth, transmission electron microscopy was applied to the nominal 2-4 nm thick catalyst layers. Fig. 7 is an example of the catalyst layer \#2 that is deposited on top of the ion conduction layer $\left(\mathrm{ZrO}_{2}\right)$. We can clearly see that the Pd layer is not continuous but rather forms small "beads." This is typical for the island growth mechanism which is often observed for metal deposition on oxides [15], especially for nonreactive metals on oxides of relatively low refractive index [16]. Images like this are important when studying the migration of Pd within the device, which is believed to be one of the main degradation mechanisms during repeated cycling.

The slowing of switching speed can be associated with a loss of catalyst, especially the catalyst next to the metal alloy, as was shown for equivalent gasochromic MnNiMg [7] and NiMg [9] switchable mirrors. Pd diffusion can be suppressed using an $\mathrm{AlO}_{\mathrm{x}}$ [6], $\mathrm{NbO}_{\mathrm{x}}$ [7] or Ti [9] (likely $\mathrm{TiO}_{\mathrm{x}}$ ) barrier layer. The idea of a barrier layer was here complemented by alloying the palladium with $9 \%$ silver. The best cycling stability to date was achieved by using a combination of thin $\mathrm{Zr} / \mathrm{ZrO}_{2}$ barrier layer and an alloyed catalyst. In Figure 8 we see a device that completed 90 cycles with an average full cycle time of 12 minutes before slowing to the point where the $30 \mathrm{~min}$. cycle timing limit became apparent. This slowing is thought to be related to loss of catalyst from critical proximity to the metal layer. The $30 \mathrm{~min}$. limit of switching caused premature reversal of the cycling polarity, leading to an apparent reduction of the switching range, as previously shown in Fig. 4.

Finally, we need to quantify the dynamic spectral reflectance in order to qualify the device as a switchable mirror. For this purpose the reflected light was collected in an integrating sphere and recorded as function of wavelength (Fig. 9). As we can see, and as 
expected, not only the transmittance is switched but the reflectance, which is of great importance to the overall energy performance of a smart window based on such switchable mirror system.

\section{Summary and outlook}

In the present paper we report on an electrochromic switchable mirror system utilizing a gas reservoir as the ion storage layer. In contrast to most other electrochromic systems which employ a solid state ion storage layer, the volume between the glass panes of an insulated glass unit is used for storing the hydrogen required for the reversible hydriding process. It was shown that the electrochromic system switches in a manner similar to that of a gasochromic system using the same magnesium alloy [7]. This was achieved by use of an optimized barrier layer consisting of a thin Zr film and a Pd catalyst alloyed with Ag. To date, about 100 full cycles can have been obtained before significant slowing was observed. Loss of catalyst function due to migration appears to be responsible for the reduction in switching speed. Future efforts need to address issues of Pd catalyst diffusion. Stresses from cyclic volume change associated with the hydriding/dehydriding processes are not currently the limiting mechanism.

\section{Acknowledgments}

We gratefully thank Sunnie Lim and Ju Lin Peng from RMIT Microscopy and Microanalysis Facility, Melbourne, Australia, for the TEM image of the Pd catalyst. We further acknowledge support by Annica Nilsson, Sam Blau, David Horwat, and Jan Isidorsson. This work was supported by the Assistant Secretary for Energy Efficiency and Renewable 
Energy, Office of Building Technology, of the U.S. Department of Energy under Contract No. DE-AC02-05CH11231. 


\section{References}

[1] C. G. Granqvist, G. A. Niklasson, and A. Azens, Appl. Phys. A: Materials Science and Processing 89 (2007) 29.

[2] J. N. Huiberts, R. Griessen, J. H. Rector, R. J. Wijngaarden, J. P. Dekker, D. G. de Groot, and N. J. Koeman, Nature 380 (1996) 231.

[3] P. van der Sluis, M. Ouwerkerk, and P. A. Duine, Appl. Phys. Lett. 70 (1997) 3356.

[4] T. J. Richardson, J. L. Slack, R. D. Armitage, R. Kostecki, B. Farangis, and M. D. Rubin, Appl. Phys. Lett. 78 (2001) 3047.

[5] T. J. Richardson, J. L. Slack, B. Farangis, and M. D. Rubin, Appl. Phys. Lett. 80 (2002) 1349.

[6] A. T. M. van Gogh, S. J. van der Molen, J. W. J. Kerssemakers, N. J. Koeman, and R. Griessen, Appl. Phys. Lett. 77 (2000) 815.

[7] J. L. Slack, J. C. W. Locke, S.-W. Song, J. Ona, and T. J. Richardson, Solar Energy Materials and Solar Cells 90 (2006) 485.

[8] J. Ell, A. Georg, M. Arntzen, A. Gombert, W. Graf, and V. Wittwer, Solar Energy Materials and Solar Cells 91 (2007) 503.

[9] S. Bao, Y. Yamada, M. Okada, and K. Yoshimura, Jap. J. Appl. Phys. 45 (2006) L588.

[10] G. Liu and T. J. Richardson, Solar Energy Materials and Solar Cells 86 (2005) 113.

[11] T. J. Richardson, J. L. Slack, and M. D. Rubin, Electrochimica Acta 46 (2001) 2281.

[12] C. G. Granqvist, A. Azens, A. Hjelm, L. Kullman, G. A. Niklasson, D. Rönnow, M. Stromme Mattsson, M. Veszslei, and G. Vaivars, Solar Energy 63 (1998) 199.

[13] V. Wittwer, A. Georg, and W. Graf, Glazing element, Patent US 6,496,295.

[14] D. R. Lide, (ed.) Handbook of Chemistry and Physics, $81^{\text {st }}$ Edition, CRC Press, Boca Raton, New York, 2000. 
[15] J. E. Greene, "Nucleation, film growth, and microstructural evolution,” in Handbook of Deposition Technologies for Films and Coatings, R. F. Bunshah, Ed., 2nd ed. Westwood, NJ: Noyes, 1994, 681-739.

[16] A. M. Stoneham, Appl. Surf. Sci. 14 (1983) 249. 


\section{Figure Captions}

Fig. 1 Schematic view (cross section) of the gas reservoir MnNiMg switchable mirror; although the layers are not exactly to scale, the relative thickness is approximately indicated. The thickest layer is the ion conductor, $\mathrm{ZrO}_{2}$, with about $0.5-1 \mu \mathrm{m}$. The second glass pane of the insulated glass unit is not shown: it is positioned above the stack at typical IGU spacing, determining the gas reservoir volume. Assuming a gas mixture of $1 \% \mathrm{H}_{2}$ and $99 \% \mathrm{Ar}$, a spacing of about $10 \mathrm{~mm}$ is required to ensure sufficient $\mathrm{H}_{2}$ in the gas reservoir.

Fig. 2 Photo of completed coating representing four devices, as seen from the glass side, before mounting in the insulated glass unit.

Fig. 3 Miniature insulated glass unit (IGU) used for testing four devices made on a single ITOcoated glass pane: each device was contacted individually via the spring "fingerstock" contacts in the frame and the spring-loaded contact touching the bus bar of the device (near the center of the IGU).

Fig. 4 Cycling tests of two devices on one glass substrate; the devices A1 and A2 were almost identical except that A1 did not have the top catalyst layer. The first 20 hours of regular cycling are not shown to better illustrate the different behaviors at this later time.

Fig. 5 Morphology of the $\mathrm{ZrO}_{2}$ ion conduction layer as seen in the scanning electron microscope: Top: “dried” $\mathrm{ZrO}_{2}$ produced by what we now consider excessive water vapor content during deposition; bottom: relatively dense $\mathrm{ZrO}_{2}$ suitable for working devices. 
Fig. 6 Optical micrograph of a damaged zone indicating partial delamination of the transparent counterelectrode (ITO); in the color version (online) one can see the interference colors along the "hills" of the structure.

Fig. 7 Transmission electron micrograph of the Pd catalyst (here catalyst layer \#2 on top of the ion conduction layer). Pd tends to grow in islands, leading to a discontinuous film. Here, no further layers were deposited after catalyst \#2, and the "glue” is from TEM sample preparation.

Fig. 8 Photopic transmittance of an optimized device; Pd catalyst migration was suppressed by using a $\mathrm{Ag}_{9} \mathrm{Pd}_{91}$ alloy (as opposed to pure $\mathrm{Pd}$ ) and the presence of a thin $(3 \mathrm{~nm})$ $\mathrm{Zr} / \mathrm{ZrO}_{2}$ barrier layer between $\mathrm{MnNiMg}$ and the $\mathrm{Ag}_{9} \mathrm{Pd}_{91}$ layer. This device showed 91 full cycles with an average full cycle period of 12 minutes, which is well within the allotted maximum 30 min to the setpoints of 20\% (upper limit) and 3\% (lower limit).

Fig. 9 Spectral reflectance of a MnNiMg device switching between metallic state and transmitting state (device similar to the one used in Fig. 8). 


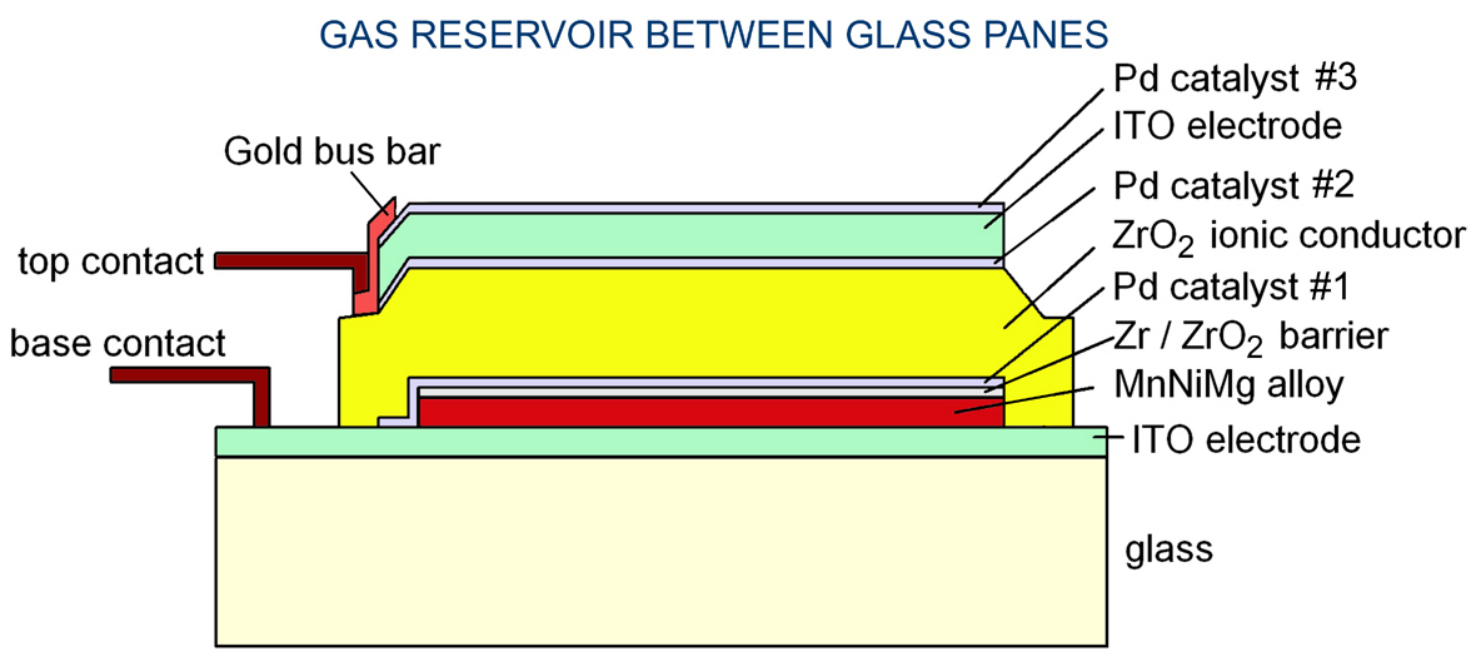

Fig. 1 


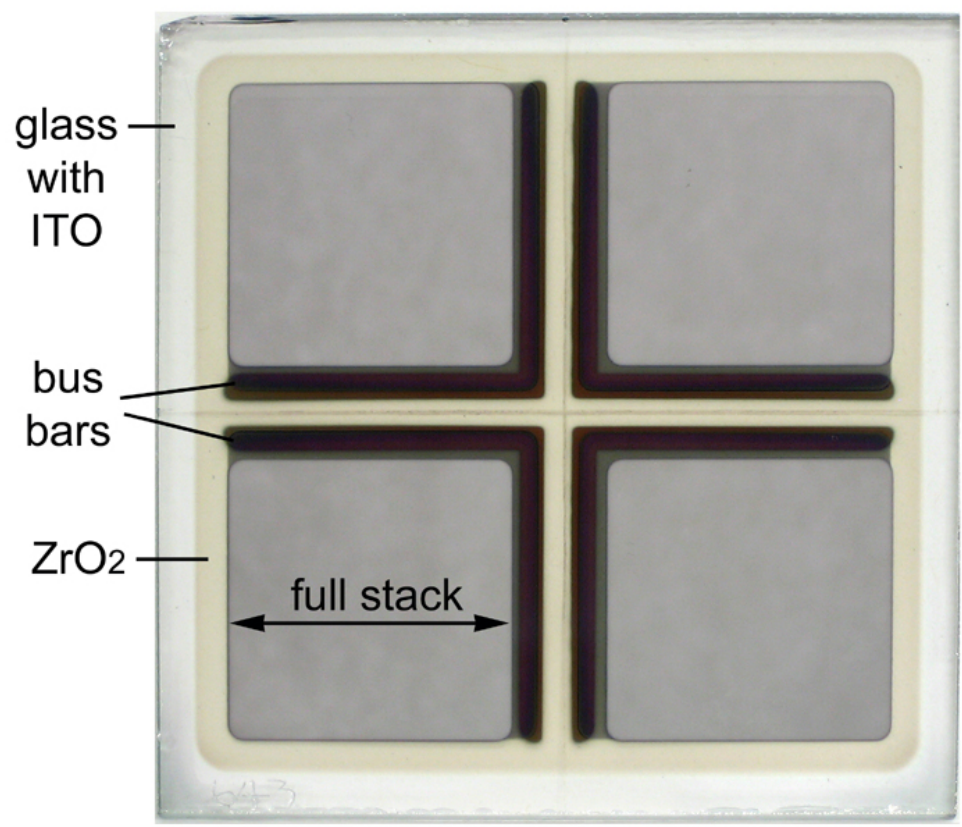

Fig. 2 


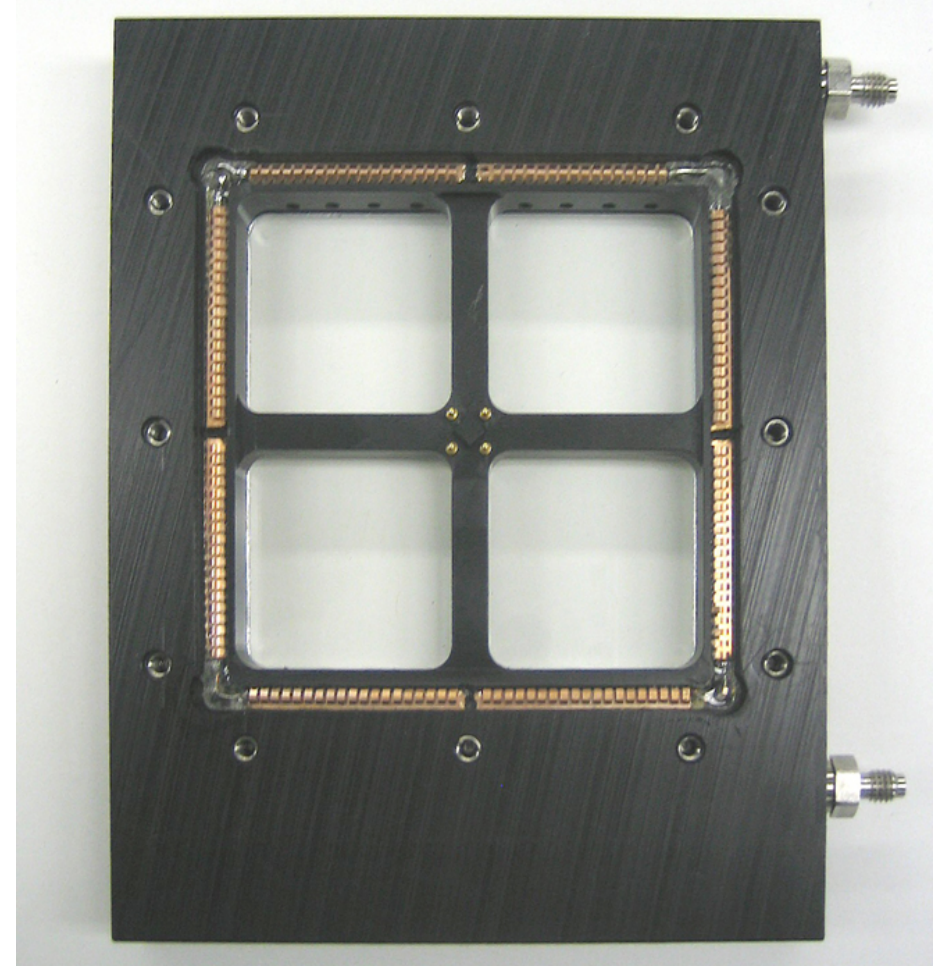

Fig. 3 


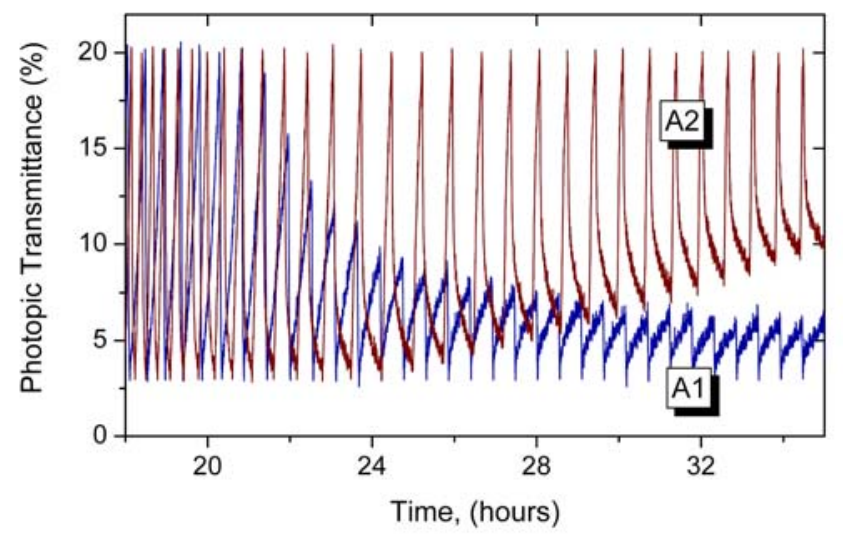

Fig. 4 


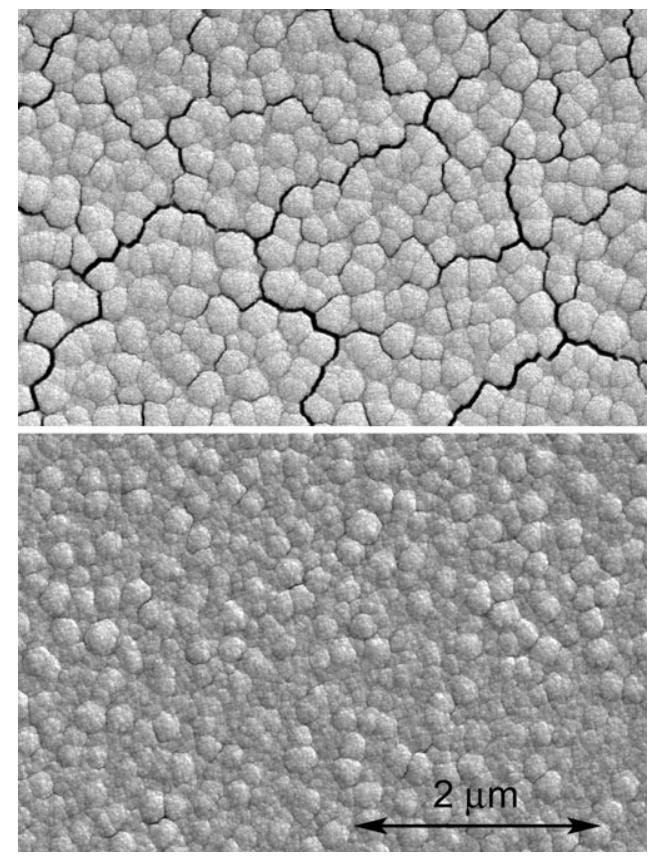

Fig. 5 


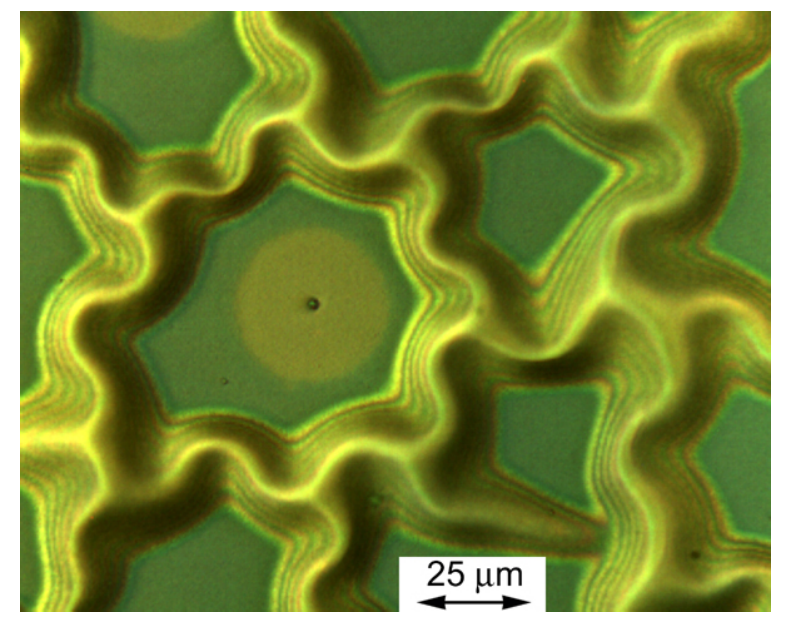

Fig. 6 


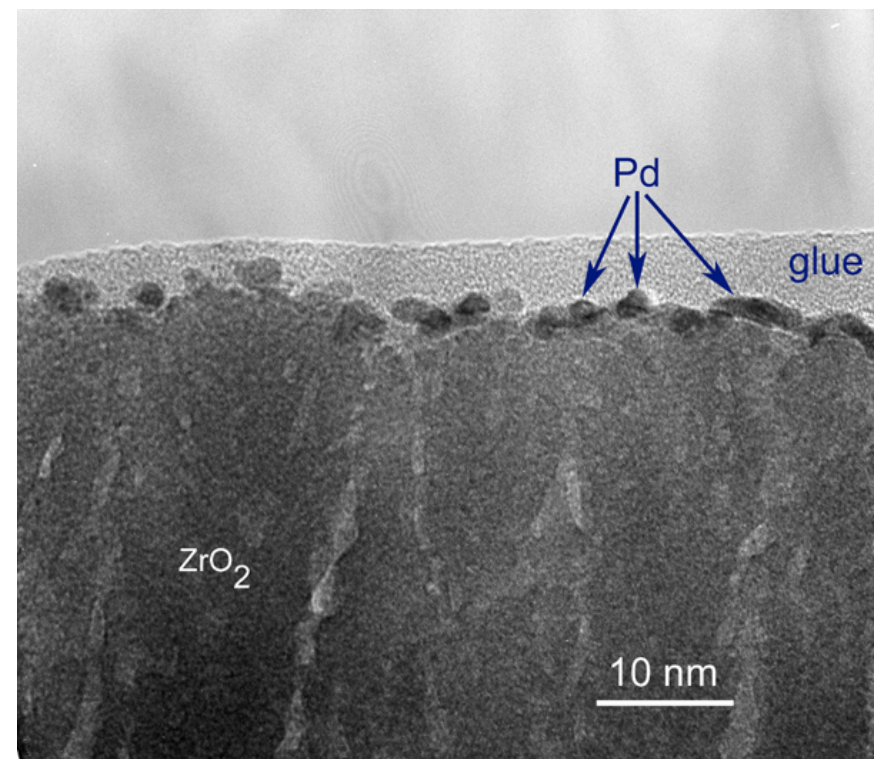

Fig. 7 


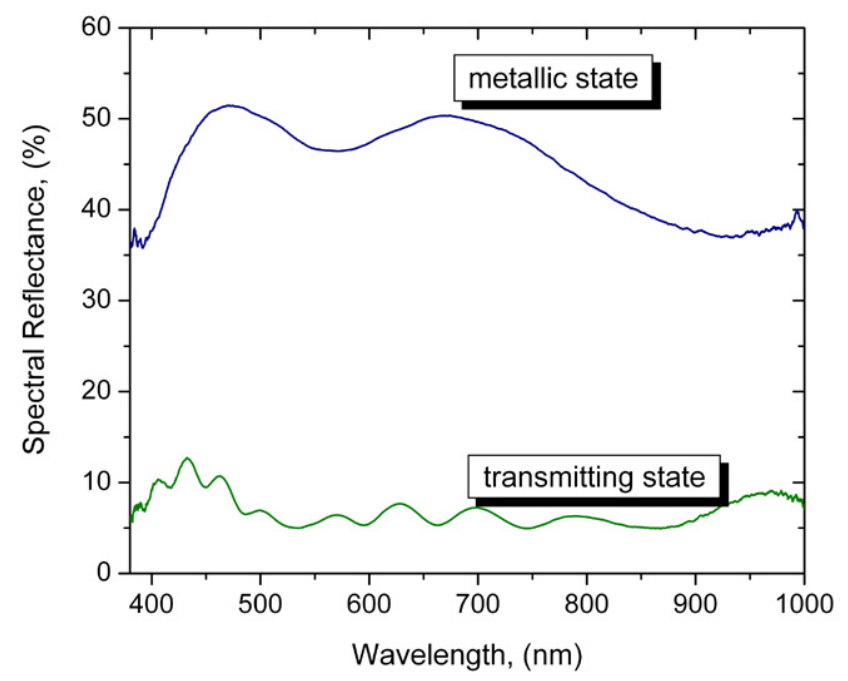

Fig. 8 
Fig. 9

Thin Solid Films, 2008 